\title{
Unmoderated Posters Basic Science, Physiology and Research
}

\begin{abstract}
UP-48
Role of Cannabinoid and TRPV1 Receptors in the Spinal Mechanisms of Micturition in the Normal Rat

Campeau, Lysanne ${ }^{1}$; Füllhase, Claudius²; Hedlund, Petter ${ }^{3}$; Howlett, Allyn ${ }^{4}$; Andersson, Karl-Erik ${ }^{5}$

${ }^{1}$ New York University, New York, NY, United States; ${ }^{2}$ Department of Urology, University Hospital Großhadern, LMU, Munich, Germany; ${ }^{3}$ Urological Research Institute, San Raffaele University, Milan, Italy; ${ }^{4}$ Department of Physiology and Pharmacology, Wake Forest University, Winston-Salem, NC, United States; ${ }^{5}$ Institute for Regenerative Medicine, Wake Forest University, Winston-Salem, NC, United States

Introduction: Systemic administration of cannabinoid (CB) receptor agonists and fatty acid amide hydrolase (FAAH) inhibitors affects bladder function, but whether the main site of action is peripheral tissues or the centra nervous system is unknown. Many of these compounds also act at the TRPV1 receptor. Our goal was to determine the effects of intrathecal (IT) CB receptor agonists and the impact of TRPV1 activation on bladder function of normal rats when spinal degradation of endogenous CBs is inhibited. Methods: Urodynamic parameters were recorded in conscious female rats at baseline, and after each drug. We first administered either IT methanandamide (MA), a selective CB1 agonist or WIN 55212-2, a non-selective CB agonist. The second part involved two other groups: the first received IT SB366791, a selective TRPV1 antagonist, followed by intraperitoneal oleoyl ethyl amide (OeTA), a FAAH inhibitor, and finally IT MA; the second group received IT vehicle (DMSO) for SB366791, followed by OeTA, and IT MA. Results: In the MA only group, bladder capacity (BC) significantly increased from baseline after $40 \mu \mathrm{g}$ administration. BC also significantly increased after administration of $40 \mu \mathrm{g}$ of WIN 55212-2 when compared to vehicle. Micturition volume (MV) increased from baseline after $20 \mu \mathrm{g}$ administration of WIN 55212-2. In the animals that received vehicle prior to OeTA and MA, we observed a significant increase from baseline in the BC and MV following both systemic OeTA and IT MA. While in the animals that were given SB366791, there were no significant changes from baseline in BC or MV following systemic OeTA and IT MA.

Conclusion: IT CB receptor agonist administration increases BC in normal rats. Both drugs may activate TRPV1 along with $C B$ receptors. IT TRPV1 antagonist administration abolished the effects of both MA and OeTA on $\mathrm{BC}$ and MV. This suggests that spinal TRPV1 activation is involved in effects by FAAH substrates and MA on afferent signaling in micturition.
\end{abstract}

\section{UP-49}

Prostate Cancer Microparticles for Follow-up After Radical Prostatectomy

Biggs, Colleen ${ }^{1}$; Al-Zahrani, Ali'; Yutkin, Vladimir'; Power, Nicholas²; Izawa, Jonathan ${ }^{2}$; Chin, Joseph ${ }^{2}$; Leong, Hon Sing 1

${ }^{1}$ Translational Prostate Cancer Research Group, London Health Sciences Centre, London, ON, Canada; ${ }^{2}$ Division of Urology, Department of Surgery, University of Western Ontario, London, ON, Canada

Background: Radical Prostatectomy (RP) is a viable option for treatment of localized Prostate Cancer (PCa). The presence of plasma-borne prostate tumour cells fragments (microparticle) is thought to correlate with the magnitude of the tumour burden. The primary objective of this study is to assess whether PCa microparticle (PCMPs) can help in the follow-up of patients after RP.

Methods: This is a prospective study to measure the PCMPs before and 3 weeks after RP. Ethics Review Board of the institution approved the trial. To identify the PCMPs population by flow cytometry, fluorophore conjugated antibodies specific for the extracellular domain of PSMA (anti-PSMA mouse IgG-RPE) and the metastasis-specific $1 \mathrm{~A} 5$ antibody (1A5 mouse IgG-FITC) were used to stain $20 \mathrm{uL}$ of plasma. Counting beads (1.0 um) were used to determine the gating parameters for identification and analysis of $1 \mathrm{~A} 5+\mathrm{PCMPs}$. We compared the total number of PSMA-positive events, 1A5+PSMA-positive events and percentage of 1A5+PSMA/total PSMApositive events before and 3 weeks after RP.

Results: In all, 25 patients were recruited for this study. Twenty-two patients had their blood tested for PCMPs before and after RP. The clinico-pathological features of these patients are summarized in Table 1. Sixteen patients $(76.1 \%)$ showed decline in the level of PCMPs after RP. The level of PCMPs did not change or increased in 6 patients (23\%). Clinical follow-up to determine if these PCa patients are at risk for persistence or recurrence of their disease is currently underway. There was no correlation between the PCMPs change status and clinico-pathological characteristic.

Conclusions: Enumeration of prostate cancer microparticles may provide a clinical means to follow patients after surgical intervention. Larger cohort and longer follow-up are needed to confirm these findings.

\section{UP-50}

\section{Involvement of Cannabinoid Receptor Type 2 in Micturition in} Mice

Campeau, Lysanne $^{1}$; Füllhase, Claudius ${ }^{2}$; Sawada, Norifumi ${ }^{1}$; Gratzke, Christian ${ }^{2}$; Hedlund, Petter ${ }^{3}$; Howlett, Allyn ${ }^{4}$; Andersson, Karl-Erik ${ }^{1}$

${ }^{1}$ Institute for Regenerative Medicine, Wake Forest University, WinstonSalem, NC, United States; ${ }^{2}$ Department of Urology, University Hospital

\begin{tabular}{|c|c|c|c|}
\hline & $\begin{array}{l}\text { All patients } \\
\quad(n=22)\end{array}$ & $\begin{array}{l}\text { Decreased } \\
\text { PCMPs } \\
\text { (n=16) }\end{array}$ & $\begin{array}{l}\text { Unchanged } \\
\text { or increased } \\
\text { PCMPs (n=6) }\end{array}$ \\
\hline Age (years) & $61.48 \pm 6.9$ & $60.6 \pm 6.4$ & $64.6 \pm 7.6$ \\
\hline $\begin{array}{l}\text { Preoperative PSA } \\
\text { (ng/dl) }\end{array}$ & $6.25 \pm 3.47$ & $6.5 \pm 3.2$ & $5.5 \pm 2.1$ \\
\hline Prostate volume (ml) & $48.28 \pm 24.9$ & $47.27 \pm 26.3$ & $55.1 \pm 24.2$ \\
\hline \multicolumn{4}{|l|}{ Biopsy Gleason } \\
\hline$\cdot 6$ & - $11(50 \%)$ & • 7 (43.7\%) & - $4(66.7 \%)$ \\
\hline$\cdot 7$ & - $9(40.9 \%)$ & • $7(43.7 \%)$ & - $2(33.3 \%)$ \\
\hline$\cdot \geq 8$ & - $2(9.1 \%)$ & - $2(12.5 \%)$ & - 0 \\
\hline No. positive biopsies & $3.04 \pm 1.98$ & $3.6 \pm 2.1$ & $2.8 \pm 1.5$ \\
\hline $\begin{array}{l}\text { Tumour greatest } \\
\text { dimension }(\mathrm{mm})\end{array}$ & $16.3 \pm 8.4$ & $19.29 \pm 7.7$ & $14.6 \pm 6.7$ \\
\hline
\end{tabular}


Großhadern, LMU, Munich, Germany; ${ }^{3}$ Urological Research Institute, San Raffaele University, Milan, Italy; ${ }^{4}$ Department of Physiology and Pharmacology, Wake Forest University, Winston-Salem, NC, United States Introduction: Systemic administration of cannabinoid (CB) receptor agonists affects bladder function, but the contribution of individual CB receptors during normal micturition has not been clearly defined. Our goal was to study if differences in urodynamic endpoints or in vitro bladder contractility exist between CB receptor type 2 knockout (CB2 -/-) and C57BL/6J control mice following CB2 receptor modulators.

Methods: After ethical approval, female C57BL/6J $(n=15)$ and CB2 -/- mice $(n=15)$ underwent bladder catheterization three days prior to cystometry. Cystometry was performed in awake animals at baseline, and after sequentially giving of HU308 (CB2 agonist) followed by AM630 (CB2 antagonist). Comparisons of effects were made with ANOVA of repeated measures. Bladders were extracted for in vitro assessment of contractility to carbachol and electrical field stimulation (EFS).
Results: Bladder capacity (BC) in C57BL/6J mice was increased from baseline by HU308, and then decreased after AM630 $(p<0.05)$. Similarly, intercontraction interval $(\mathrm{ICI})$ increased from baseline with $\mathrm{HU} 308$, and then decreased with AM630.

CB2 -/- mice had at baseline a lower maximal pressure (MP), basal pressure $(B P)$, and area under the curve (AUC), and a higher $\mathrm{ICl}, \mathrm{BC}$ and compliance than controls $(p<0.05)$. AM630 in CB2 -/- mice increased ICl, BC, MP and TP, and decreased BP $(p<0.05)$. There were no differences in contractility after carbachol or EFS between both groups.

Conclusion: Indicating a role for the CB2 receptor in afferent micturition signals in normal mice, CB2 receptor agonism modified $\mathrm{ICI}$ and BC. CB2 -/- mice had lower pressures, along with a longer $\mathrm{ICl}$ and larger BC and compliance than $\mathrm{C} 57 \mathrm{BL} / 6 \mathrm{~J}$. These differences may be explained by compensatory up-regulation of other signals due to the knockout of CB2. Further investigations are necessary to understand the reverse effects by AM630 in CB2-/- and control mice.

\section{CUA ABSTRACTS}

\section{Unmoderated Posters Education}

\begin{abstract}
UP-51
Case-based Educational Approach to Improve Interprofessional Interaction in the Management of Patients with Advanced Prostate Cancer

Saad, Fred'; Lukka, Himanshu²; So, Alan ${ }^{3}$; Emmenegger, Urban4; AudetLapointe, Marika'; Meisner, Janice ${ }^{5} ;$ St. Germain, France $^{6}$

${ }^{1}$ University of Montreal, Montreal, QC, Canada; ${ }^{2}$ McMaster University, Hamilton, ON, Canada; ${ }^{3}$ UBC, Vancouver, BC, Canada; ${ }^{4}$ University of Toronto, Toronto, ON, Canada; ${ }^{5}$ Novapoint, Montreal, QC, Canada; ${ }^{6}$ Oncology, Sanofi Canada, Laval, QC, Canada

Introduction and Objective: Research advances in castration-resistant advanced prostate cancer have resulted in the availability of treatment options which can prolong life \& improve its quality. With these advancements, long-term patient management necessitating a strong knowledge base of treatment options as well as on-going, effective communication among interprofessional healthcare providers is required to support positive patient outcomes.
\end{abstract}

Methods: ProstAIDE (Prostate Cancer Action, Information \& Education) was developed as an educational initiative for this interprofessional group to educate team members on the global management of patients with advanced prostate cancer. A faculty of prostate cancer specialists was formed to develop components to address needs with program materials $\&$ tools. Identified areas of unmet educational need include interprofessional communication gaps \& optimization of referral between providers. Results: The ProstAIDE program resulted in the development of a casebased, interactive program. Module 1 emphasizes the interprofessional care of patients using case studies to illustrate the appropriate timing of referral from one specialty to another. Module 2 focuses on disease management using case studies to illustrate treatment optimization \& the management of psychological health of patients. Interactive questions were included throughout both modules to identify \& highlight practice issues experienced by clinicians. Additionally, a ProstAIDE patient resource kit was developed to support patients in discussions with their interprofessional healthcare providers. To date, 4 sessions have been completed with positive evaluations \& requests for more programs.

Conclusion: ProstAIDE has proven its importance in supporting education for the interprofessional healthcare team responsible for the care of advance prostate cancer patients. Additional programming has been scheduled to support ongoing \& interprofessional education \& collaboration. 